\title{
License Plate Character Recognition using Riesz Fractional and Convolutional Neural Network
}

\author{
Challa Chaitanya Chowdary, Udaya Kiran
}

\begin{abstract}
Automatic license plate recognition system is mostly used for identification of vehicles. This system is used in traffic monitoring, parking management and identification of theft vehicles. As in India the license plate regulations are not strictly followed, it is often difficult to identify the plate with different font type and character size. One more major problem in license plate recognition is low quality of images which affected via severe illumination condition. In this paper, a Riesz fractional mathematical model is proposed for enhancing the edges, which results in improving the performance of text recognition. The text in the license plate is recognized using the convolution neural network and the results showed better accuracy.
\end{abstract}

Keywords: Character recognition, convolutional neural network, license plate recognition.

\section{INTRODUCTION}

Automatic license plate recognition system plays a vital role in a real-time environment like traffic monitoring, toll collection and parking management. Image processing and neural networks place a major role in the recognition of characters from the license plate. As the system is a real-time application, high detection and recognition algorithms are required. This system is mainly affected by factors like severe outdoor illumination conditions, effects from headlight and sunlight, low-quality license plate images and perspective distortions. In this paper Riesz fractional mathematical model is used for enhancing the edge details in the image and a convolution neural network $\mathrm{CNN}$ is used for character recognition.

Abolghasemi and Ahmadyfard [1] proposed enhancing methods based on intensity variance and edge density to increase the contrast of license plate regions. In the proposed model, the number plates are detected by using the colored texture properties. The MNS (multimodal neighborhood signature) method was used for characterizing the information in the detected number plate. But the proposed model is undesirable for real-time applications as it is highly complex. Baohua and Lanying [2] proposed an algorithm for detecting number plate location based on histogram equalization.
To enlarge the intensity difference among objects and background, the traditional algorithm was improved by using histogram equalization. Ghaili et al. [3] proposed a fast method for car-license plate detection (CLPD) using vertical edge detection algorithm (VEDA) based on the contrast between the grayscale values. Azm and Islam [4] proposed a new automatic license plate detection method based basic enhancement techniques, which effectively detected license plate area from an image in the hazardous conditions. However, it was observed that the proposed system was effective only in removing the rain approach from the car images. Vicente and Pedrini [5] proposed a very low quality license plate image recognition using learning-based single-image super-resolution method. The low quality images were reconstructed into high quality images by enhancing the perceived details and sharpness of the images using super-resolution method. Yu et al. [6] proposed a robust method based on wavelet transform and empirical mode decomposition(EMD) analysis for searching the license plate location. To enhance the edge details of texts, histogram equalization was used. However, the proposed method works only under restricted conditions such as fixed illumination, limited vehicle speed, designated routes, and stationary backgrounds.

Saha et al. [7] proposed Integrated Traffic Management System (ITMS) for India. In the proposed system the binary images were obtained using sobel gradient information. The characters were recognized by neural network using the features extracted. Binarization and grouping performed by Hough transformation had impact on the performance of the model. Guo et al. [8] proposed a license plate recognition (LPR) model based on extremal regions and restricted Boltzmann machines. Two different localizations methods on color information and texture of the license plates were proposed. Tian et al. [9] proposed a two-stage character segmentation method for Chinese license plate. The characters in the license plate were identified with a template using harrow-shaped filter bank and minimum response. A path finding algorithm was used to segment the connected and overlapped characters.

Based on the literature survey, that was observed that most of the research was on image recognition rather than image enhancement. As image enhancement places a major role in recognition of a new model for low-quality images using Riesz fractional is proposed. A 
convolution neural network was used for recognition.

\section{METHODOLOGY}

The input to the proposed ANPR system is the video recorded using a digital camera. Image acquisition is the first step of the recognition system, where the images of the vehicles are captured using a digital camera placed at a fixed location.

After the acquisition, pre-processing plays a major role in image analysis. In the acquired frames there are changes of noises to be present. Figure 1 shows the complete pre-processing steps.

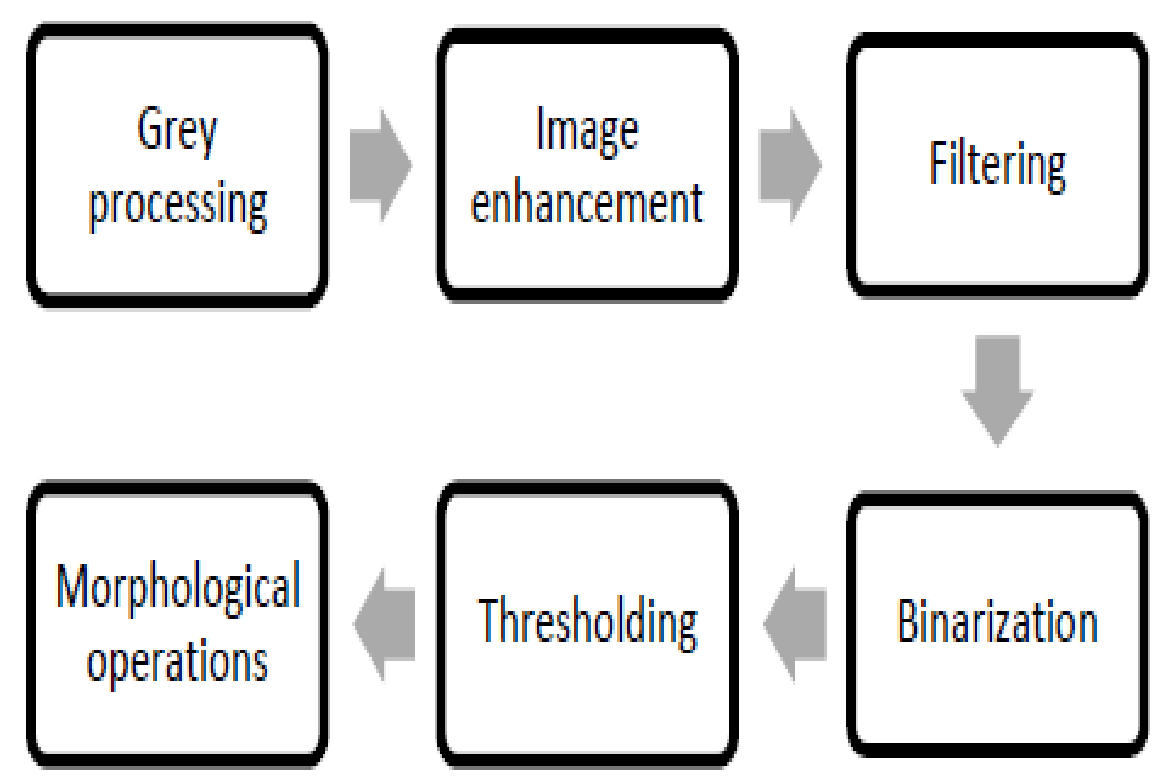

Figure 1: Steps for Image Pre-Processing

The captured color frames are to be converted into gray scale frame. The color to grayscale conversion is performed by Equation 1.

$$
\text { Gray }=(0.299 \times R)+(0.587 \times G)+(0.114 \times B)----(1
$$

\section{Image Enhancement}

The principal objective of the image enhancement is to process an image for a better view than the original image. The images acquired from a device are prone to various errors like spatial and temporal limitations, which results in noise, bad illumination, and blur in the acquired images.

\section{Filtering}

The image enhancement is performed to enhance the low-frequency details in the regions where gray-level changes are insignificant using the Riesz fractional. As the proposed model emphasizes edges in images by the convolution between Riesz mask and the input image. Riesz Fractional derivative nonlinearly preserves both edge features in image regions where the gray level changes extensively, and texture features in other regions where the gray level does not change extensively [10]. For the input image $I$, the enhanced image $I_{h}$ is calculated using the Equation 2. Where $\mathrm{W}$ is the window mask calculated using the coefficients $(w 1, w 2, \ldots, w n)$.

$$
I_{h}(v, \lambda)=W \times I(v, \lambda)
$$

These coefficients define the masks which are denoted by $\mathrm{W}$ as shown in Equation 3.

$$
W=w_{2} w_{1} w_{2} w_{1} w_{0} w_{1} w_{2} w_{1} w_{2}
$$

The coefficients of Riesz Fractional derivative of order $\alpha$ are calculated using Equations 4-7.

$$
\begin{gathered}
w_{0}=\frac{2 \pi(\alpha+1)}{\pi\left(\frac{\alpha}{2}+1\right)^{2}} \\
w_{1}=\frac{(-2) \pi(\alpha+1)}{\pi\left(\frac{\Omega}{2}\right) \pi\left(\frac{\pi}{2}+2\right)^{2}}-\cdots \\
w_{2}=\frac{2 \pi(\alpha+1)}{\pi\left(\frac{\pi}{2}-1\right) \pi\left(\frac{\alpha}{2}+a\right)^{2}} \cdots \cdots \\
w_{n}=\frac{2(-1)^{n} \pi(\alpha+1)}{\pi\left(\frac{\alpha}{2}-n+1\right) \pi\left(\frac{\pi}{2}+n+1\right)^{2}}
\end{gathered}
$$

\section{Binarization}

The image with various grey level intensities are converted into a binary image to highlight the foreground and suppress the background using the Otsu's method. The license plate image with size $M * N$ is defined as $f(x, y)$, where $0 \leq x<M$ and $0 \leq y<N$. The gray level of the image $f(x, y)$ is converted into a binary image using Equation 8.

$f\left(x_{v} y\right)=\left\{1_{v}\right.$ if $f(x, y)>$ Th 0, if $f(x, y) \leq T h$

Th is the threshold, computed as shown in Equation 9.

$$
T h=A \times \sum_{i=0}^{m} \quad \sum_{j=0}^{n} \quad \frac{\operatorname{Grayr}(i, j)}{M \times N}
$$

Where, $\operatorname{Gray}(i, j)$ is the gray value of the pixel and the background of the license plate image determines the coefficient of $\mathrm{A}$. 


\section{Morphological Operations}

Morphological operations are performed to remove unwanted small objects and the holes are filled. The number plate consists of small lines and dots; these are considered as small regions. These regions create problems while segmentation and recognition. So these regions are identified and removed from the image.

\section{Character segmentation}

The characters and numbers from the number plate are separated in order to identify them. The characters/ numbers are segmented from the binary image by extracting connected components.

\section{Recognition}

In order to recognize the segmented characters efficiently, convolution neural network was used. Convolution neural network mainly consists of five layers namely an input layer, a convolution layer, a pooling layer, a fully connected layer, and an output layer as shown in Figure 2. CNN operates in two stages - feature learning stage and a classification stage. The feature learning stage provides the most significant features by combining convolution layers and pooling layers [11].

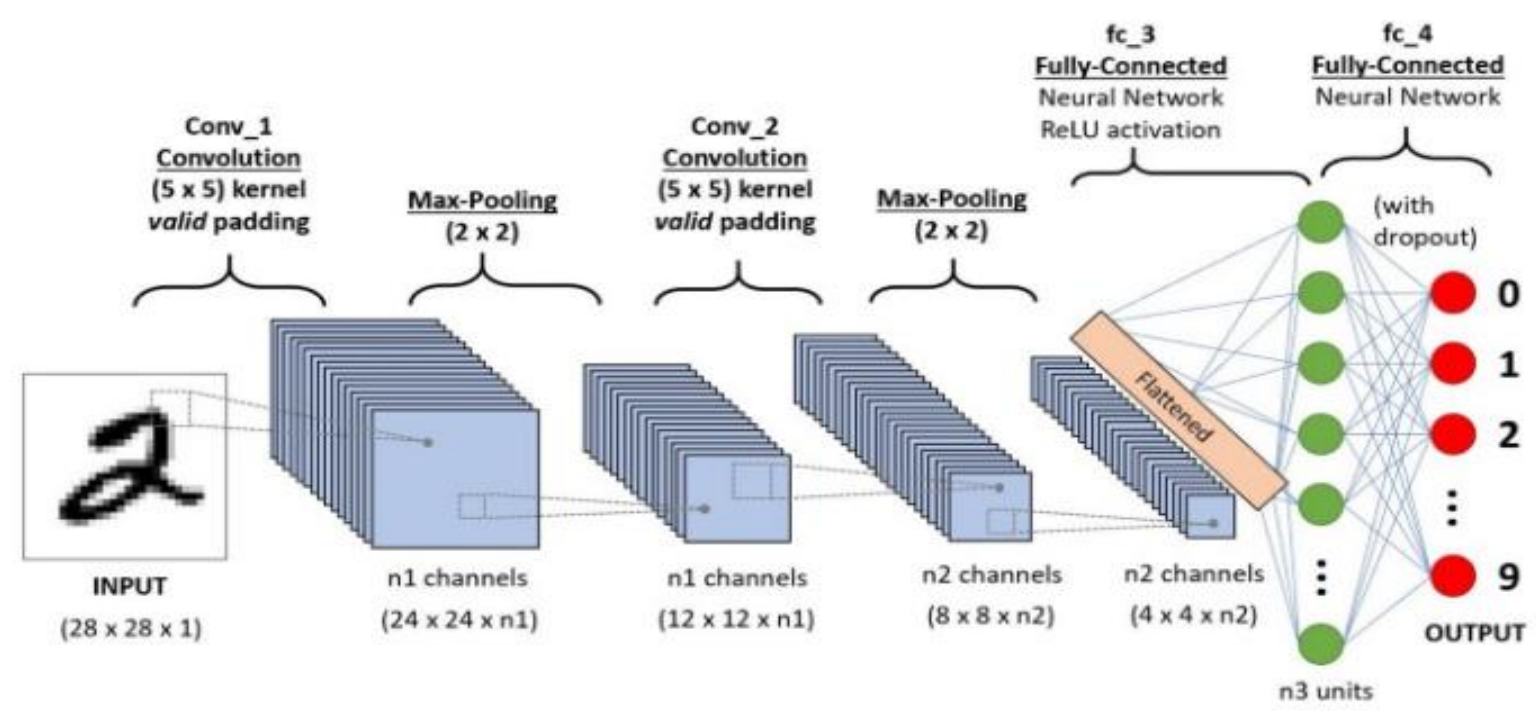

Figure 2: Convolution neural network model

The character/ number extracted from number plate is provided as input to the $\mathrm{CNN}$ input layer. In this layer padding is added to the image to ensure that no subset is incomplete. A set of filters were used over the input layer in the convolution layer. The results of each subset are then mapped to one single point and these calculations are repeated on the entire image. After completion of this process the output of the convolution layer is provided as input to polling layer. In order to make the calculations easier, the size of the input is reduced in pooling layer. In the proposed model the size is reduced by half. The pooling layer consists of filters of sizes $2 \times 2$ that takes a subset of 4 pixels and deduces them to one pixel having the highest value of that subset; This final decision is implemented in the fully connected layer. The output of the last pooling layer is provided as input to this layer. The output layer has a vector of 10 neurons, one for each possible digit outcome.

\section{RESULT AND DISCUSSION}

In order to examine the performance of the proposed model, a real time video from a toll gate has been collected. The video has captured in night conditions to check the overall performance. Initially, CNN was trained with 62992 synthesized characters from computer fonts. As the Indian number plates are made up of different fonts based on the customer requirement. So the $\mathrm{CNN}$ is trained with different font images of size $28 \times 28 \times 1$. The overall dataset is portioned into 70:30 ratios for training and testing the model. The CNN model is shown in Figure 3. A GUI for the proposed model was developed as shown in Figure 4. This GUI provides the original frame, enhanced frame, segmented license plate and finally the recognized values of the number plate. The overall performance of the $\mathrm{CNN}$ model is evaluated using RMSE, PSNR, SSIM, recall, precision, F-measures as shown in Table 1. From the results it is clear that the proposed model gave better performance. 


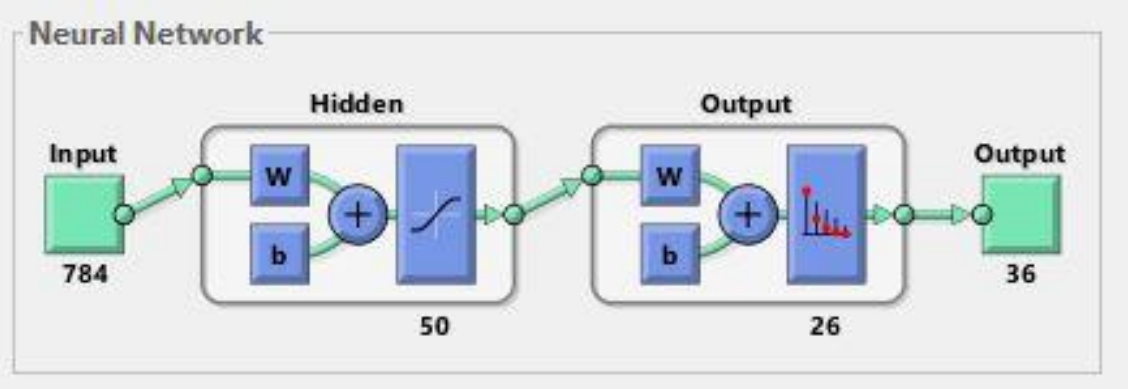

Figure 3: CNN model

\section{Orginal Frame}

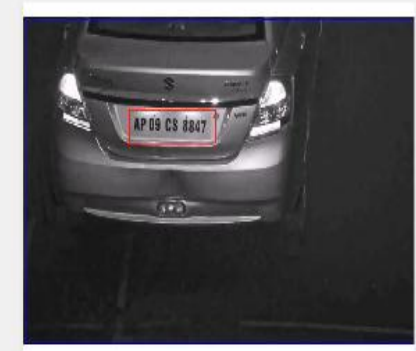

\section{Number plate}

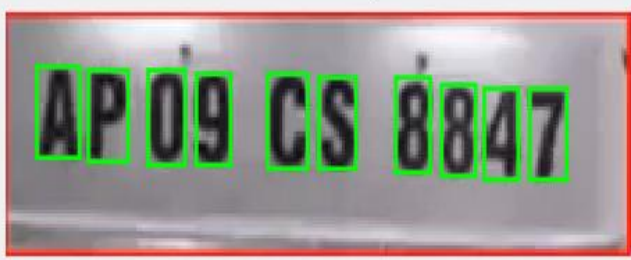

\section{Enchanced Frame}

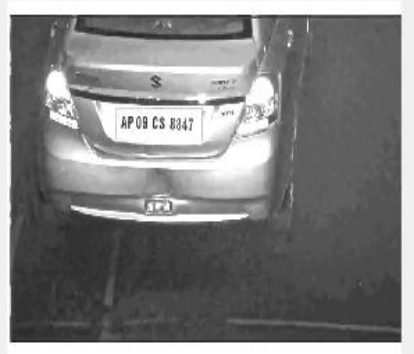

\section{AP09CS8847}

Figure 4: GUI of the proposed model

Table 1: Performance analysis of the proposed model

\begin{tabular}{|c|c|}
\hline Parameters & Proposed Method \\
\hline RMSE & 3.8458 \\
\hline PSNR & 28.3002 \\
\hline SSIM & 0.9255 \\
\hline RECALL & 96.41 \\
\hline PRECISION & 96.43 \\
\hline F-MEASURES & 96.42 \\
\hline
\end{tabular}

\section{CONCLUSION}

The vehicles are identified using the license plate. So automatic license plate recognition system plays a major role in traffic monitoring, parking management and identification of theft vehicles. As in India the license plate regulations are not strictly followed, it is often difficult to identify the plate with different font type and character size. One more major problem in license plate recognition is low quality of images which affected via severe illumination condition. It is also noted 
that image quality especially license plate images get affected by multiple factors, such as low contrast, non-uniform illumination, perspective distortion, camera movement, the speed of the vehicle, headlight, sunlight, rain, fog, and weather. In this paper, a Riesz fractional mathematical model is proposed for enhancing the edges, which results in improving the performance of text recognition. The CNN model was used for recognizing the characters/numbers in the plate and it was observed that the complete proposed model showed better accuracy.

\section{REFERENCES}

1. Abolghasemi, V. and Ahmadyfard, A., 2009. An edge-based color-aided method for license plate detection. Image and Vision Computing, 27(8), pp.1134-1142.

2. Baohua, Z., Dahua, Y., Hongmei, H. and Lanying, G., 2010, June. License plate location algorithm based on histogram equalization. In 2010 International Conference On Computer Design and Applications (Vol. 1, pp. V1-517). IEEE.

3. Al-Ghaili, A.M., Mashohor, S., Ramli, A.R. and Ismail, A., 2012. Vertical-edge-based car-license-plate detection method. IEEE transactions on vehicular technology, 62(1), pp.26-38.
4. Azam, S. and Islam, M.M., 2016. Automatic license plate detection in hazardous condition. Journal of Visual Communication and Image Representation, 36, pp.172-186.

5. Vicente, A.N. and Pedrini, H., 2016, October. A learning-based single-image super-resolution method for very low quality license plate images. In 2016 IEEE International Conference on Systems, Man, and Cybernetics (SMC) (pp. 000515-000520). IEEE.

6. Yu, S., Li, B., Zhang, Q., Liu, C. and Meng, M.Q.H., 2015. A novel license plate location method based on wavelet transform and EMD analysis. Pattern Recognition, 48(1), pp.114-125.

7. Saha, S., Basu, S. and Nasipuri, M., 2015. iLPR: an Indian license plate recognition system. Multimedia Tools and Applications, 74(23), pp.10621-10656.

8. Gou, C., Wang, K., Yao, Y. and Li, Z., 2015. Vehicle license plate recognition based on extremal regions and restricted Boltzmann machines. IEEE Transactions on Intelligent Transportation Systems, 17(4), pp.1096-1107.

9. Tian, J., Wang, R., Wang, G., Liu, J. and Xia, Y., 2015. A two-stage character segmentation method for Chinese license plate. Computers \& Electrical Engineering, 46, pp.539-553.

10. Raghunandan, K.S., Shivakumara, P., Jalab, H.A., Ibrahim, R.W., Kumar, G.H., Pal, U. and Lu, T., 2017. Riesz fractional based model for enhancing license plate detection and recognition. IEEE Transactions on Circuits and Systems for Video Technology, 28(9), pp.2276-2288.

11. Jain, V., Sasindran, Z., Rajagopal, A., Biswas, S., Bharadwaj, H.S. and Ramakrishnan, K.R., 2016. Deep Automatic Licence Plate Recognition system. 